\title{
A Call to Action: Novel Ways that Hepatologists Can Contribute to Patient Care During the COVID-19 Pandemic
}

\author{
Patricia P. Bloom ${ }^{1}$ \\ Published online: 13 April 2020 \\ ○) Springer Science+Business Media, LLC, part of Springer Nature 2020
}

When hepatologists first learned about COVID-19 in December 2019, we felt distanced from the infection geographically, but also in terms of our specialty. The COVID19 pandemic felt wholly in the purview of infectious disease. Over a matter of months, that distance has narrowed considerably: certainly, in terms of geographic separation as the infection has now been reported in every state in the USA and numbers swell at our institution, but also with respect to our specialty. We have found several ways in which hepatologists can play an important role in the pandemic (Table 1):

1. Consultation regarding elevated liver enzymes in patients with COVID-19: Up to 50\% of patients infected with COVID-19 develop abnormal elevations of liver enzymes [1,2]. Many patients have no prior liver evaluation, and it remains uncertain if they have a primary hepatic condition or the liver enzymes are elevated in association with COVID-19 infection or due to druginduced liver injury. Some providers may instinctively evaluate these abnormal enzymes with a hepatobiliary ultrasound, but this could place radiology technicians at unnecessary risk for COVID-19 exposure and use stores of valuable personal protective equipment (PPE). Similarly, hepatitis B or C viral PCRs could be performed, which, depending on the hospital's COVID19 testing strategy, could impede the hospital's ability to run COVID-19 PCRs. We have found it valuable to review specific COVID-19 cases with our hepatology group and create uniform, yet dynamic, guidance around how to evaluate (or defer evaluation) of abnormal liver enzymes during the pandemic. We have also developed an efficient e-consultation service with a templated and evolving note, which facilitates rapid feedback to infec-

Patricia P. Bloom

ppbloom@partners.org

1 Division of Gastroenterology, Massachusetts General Hospital, Boston, MA 02114, USA tious disease specialists and medicine colleagues and spares PPE utilization while limiting patient-physician exposure. Many of these patients can defer detailed liver evaluation during the pandemic.

2. Management of COVID-19 therapies in patients with elevated liver enzymes: Many of the medications being used to treat patients with COVID-19, including as part of therapeutic trials, can be associated with liver enzyme elevation. Some examples include acetaminophen, statins, remdesivir, and antibiotics. Hepatologist participation in multi-disciplinary rounds provides perspective and guidance regarding the severity and etiology of elevated liver enzymes, in some cases influencing therapeutic management decisions.

3. Liver transplant management: The COVID-19 pandemic will influence liver transplantation in numerous ways. Liver transplant providers will be increasingly at risk of exposure and infection with COVID-19, through ongoing interaction with the health care system. Liver transplant recipients will likewise be exposed and risk severe infection with COVID-19 in the post-transplant period. Other resources essential to liver transplantation such as operating rooms, intensive care unit beds, ventilators, and PPE will become more challenging to consistently secure. Finally, the donor pool will increasingly become infected with COVID-19, limiting safe graft options. Hepatologists can provide active support to their liver transplant programs by creating new protocols to optimize safety and efficacy of transplant during this uncertain time.

4. Management of cirrhosis when decompensated by COVID-19 infection: As the transplant list wait time increases, hepatologists will be called upon to manage an increasing volume of advanced cirrhosis. Patients with cirrhosis will be prone to decompensation if infected with COVID-19, and hepatologists will be called on to weigh the risks versus benefits of certain standard practices, such as a diagnostic paracentesis, 
Table 1 Contributions that hepatologists can make toward patient care during the COVID-19 pandemic

\begin{tabular}{|c|c|}
\hline Clinical issues & Description \\
\hline Consultation regarding elevated liver enzymes & $\begin{array}{l}\square \text { Determine urgent versus non-urgent evaluation of abnormal liver enzymes } \\
\square \text { Defer non-urgent evaluation and create systems for future evaluation } \\
\square \text { Develop systems within each hepatology practice to provide up-to-date, uniform and yet } \\
\text { dynamic guidance on liver enzymes in COVID-19 pandemic }\end{array}$ \\
\hline $\begin{array}{l}\text { Management of COVID-19 therapies in } \\
\text { patients with elevated liver enzymes }\end{array}$ & $\begin{array}{l}\square \text { Provide guidance in multi-disciplinary teams around severity and etiology of liver enzyme } \\
\text { elevations } \\
\square \text { Assist in therapeutic management decisions as it relates to potential hepatotoxicity }\end{array}$ \\
\hline Liver transplantation management & $\begin{array}{l}\square \text { Create and iterate protocols to optimize safety and efficacy of liver transplant given evolving } \\
\text { constraints }\end{array}$ \\
\hline $\begin{array}{l}\text { Decompensated cirrhosis management in } \\
\text { patients infected with COVID-19 }\end{array}$ & $\begin{array}{l}\square \text { Manage the growing list of patients with decompensated cirrhosis as transplant wait time } \\
\text { prolongs } \\
\square \text { Weigh new risks versus benefits of previously standard practices, such as diagnostic paracen- } \\
\text { tesis and endoscopy }\end{array}$ \\
\hline Assistance with the general medicine service & $\square$ Provide much needed services to general medicine \\
\hline
\end{tabular}

which may now pose risks of exposure to health care workers.

5. Finally, as hospital volumes surge and health care workers fall ill, we will be called upon to return to our roots and practice all forms of medicine.

The pathway forward demands strong multi-disciplinary efforts and fastidious administrative effort. Hepatology practices should remain up to date with rapidly evolving literature and forge open lines of communication within their group to maintain a shared understanding of evolving best practices. Finally, hepatologists should collect data in a rigorous fashion about the outcomes in this era, so our community can quickly adapt our clinical practice in a scientific manner.

\section{References}

1. Xu L, Liu J, Lu M, Yang D, Zheng X. Liver injury during highly pathogenic human coronavirus infections. Liver Int. 2020.

2. Fan Z, Chen L, Li J, et al. Clinical features of COVID-19 related liver damage. medRxiv. 2020:2020.02.26.20026971.

Publisher's Note Springer Nature remains neutral with regard to jurisdictional claims in published maps and institutional affiliations. 